\title{
Laboratory and Imaging Evaluation of Cardiac Involvement in Patients with Post-Acute COVID-19
}

\author{
Ersin Sarıçam, (D) ${ }^{1,2}$ \\ Ali Doğan Dursun, (D) ${ }^{3,4}$ \\ Gülçin Türkmen Sarıyıldız, ${ }^{5,6}$ \\ Nalan Can, ${ }^{7}$ Engin Bozkurt, ' \\ Uğur Gönüllü, ${ }^{8}$ Nihal Basay, \\ Meral Türkmen, ${ }^{10}$ \\ Abdülaziz Denli, ' Mustafa Ünlü' \\ 'Department of Cardiology, Medicana \\ International Ankara Hospital, Ankara, \\ Turkey; ${ }^{2}$ Department of Cardiology, \\ Atılım University Medical School, Ankara, \\ Turkey; ${ }^{3}$ Department of Physiology, \\ Atılım University Medical School, Ankara, \\ Turkey; ${ }^{4}$ Check-up Center, Home Care \\ Services, Medicana International Ankara \\ Hospital, Ankara, Turkey; ${ }^{5}$ Department of \\ General Surgery, Medicana International \\ Ankara Hospital, Ankara, Turkey; \\ ${ }^{6}$ Operating Room Services, Vocational \\ School of Health Services, Atılım \\ University, Ankara, Turkey; ${ }^{7}$ Department \\ of Nuclear Medicine, Medicana \\ International Ankara Hospital, Ankara, \\ Turkey; ${ }^{8}$ Department of Pulmonary \\ Medicine, Atılım University Medical \\ School, Ankara, Turkey; ${ }^{9}$ Department of \\ Pulmonary Medicine, Medicana \\ International Ankara Hospital, Ankara, \\ Turkey; ${ }^{10}$ Department of Internal \\ Medicine, Medicana International Ankara \\ Hospital, Ankara, Turkey; "'Department \\ of Physical Medicine and Rehabilitation, \\ Medicana International Ankara Hospital, \\ Ankara, Turkey; ${ }^{12}$ Department of \\ Nuclear Medicine, Gazi University \\ Medical School, Ankara, Turkey
}

Correspondence: Ersin Sarıçam Department of Cardiology, Medicana International Ankara Hospital, Söğütözü Cd. 2165. Sokak No: 6, Söğütözü, Ankara, 06520, Turkey

Tel +905324066440

Fax +903122203170

Email saricamersin@yahoo.com
Background: In the post-acute COVID-19 syndrome, many patients suffer from palpitations, effort-associated fatigue, and even sudden death. The mechanism of heart involvement in this syndrome is uncertain. The main purpose of the study was to identify possible cardiac involvement causes in patients with post-acute COVID-19 by using biomarkers such as NTproBNP and nitric oxide (NO) and cardiac imaging modalities.

Methods: In this cross-sectional study, a total of 105 participants were included according to the existence of symptoms, and 40 of these participants were asymptomatic patients. The ages of the participants ranged from 20 to 50 years. All patients were healthy before COVID19. The symptoms were defined as palpitations and/or fatigue association with exercise in post-acute COVID-19 term. The comparison of the two groups was made by using biochemical parameters (NT-proBNP, Troponin I, NO) and imaging techniques (echocardiography, cardiovascular magnetic resonance $(\mathrm{CMR})$ and cardiac positron emission tomography (PET)).

Results: The symptomatic patients had higher NT-proBNP levels compared with asymptomatic patients $(132.30 \pm 35.15 ; 76.86 \pm 16.79$, respectively; $\mathrm{p}<0.001)$. Interestingly, the symptomatic patients had lower NO levels than asymptomatic patients $(9.20 \pm 3.08 ; 16.15$ \pm 6.02 , respectively; $\mathrm{p}<0.001)$. Echocardiography and CMR were normal. However, we found regional increased 18F-FDG uptake on cardiac PET to be compatible with myocardial fatigue.

Conclusion: We found elevated NT-proNBP levels, low serum NO levels, and increased 18F-FDG uptake on cardiac PET in post-acute COVID syndrome. Cardiac PET could replace or be added to CMR for detecting subtle subacute/chronic myocarditis. The follow-up of patients with post-acute COVID-19 could target the possibility of risk of heart failure.

Keywords: post-acute COVID-19, cardiac involvement, laboratory tests, imaging

\section{Introduction}

Coronavirus disease of 2019 (COVID-19) is a deadly pandemic infection. ${ }^{1}$ Although the primary mortality reason in COVID-19 is severe acute respiratory syndrome, cardiovascular complications of COVID-19 have been described as acute coronary syndrome, myocarditis, arrhythmia, and thromboembolism in early terms. ${ }^{2}$ On the other hand, after the acute COVID-19 period, a group of patients have suffered from palpitations, chest pain, and effort-associated fatigue, which has been described as post-acute COVID-19 syndrome. ${ }^{3}$ These patients have also common arrhythmias. ${ }^{4}$ Besides, comorbid conditions including heart failure and 
diabetes in those patients increase re-hospitalization or death. ${ }^{5}$ However, the patients without comorbid situations have normal troponin level, normal electrocardiographic findings in addition to normal pulmonary functions.

Cardiac biomarkers have been used for cardiac damage establishment in recent years. N-terminal pro B-type natriuretic peptide (NT-proBNP) is a well-known biomarker originating from cardiomyocytes. These biomarkers have important diagnostic and prognostic values at earlystage and asymptomatic cardiovascular diseases. ${ }^{6,7}$ Interestingly, it has been stated that a NT-proBNP test could predict as an independent risk factor for in-hospital death in patients with severe COVID-19. ${ }^{8}$ Recent studies reported that vascular problems in acute COVID-19 could be related with lower nitric oxide levels. ${ }^{9}$ To date, there is no comprehensive examination of the potential cardiac involvement mechanism in post-acute COVID-19 syndrome. Pericardial effusion availability, cytokine induction and intracardiac virus detection can be very important in terms of cardiac involvement of COVID-19.

To understand the likely basis of the cardiac involvement in post-acute COVID syndrome, we evaluated recovered COVID-19 patients using laboratory and imaging investigation techniques.

\section{Method}

The study population consisted of 105 patients who had recovered from COVID-19 between December 2020 and February 2021. This study was a cross-sectional study. The age range of the groups was from 20 to 50 years. This study comprised patients having recovered from COVID19 from 3 weeks to 2 months ago and with no prior heart disease. The study protocol was accepted by Medicana International Ankara Hospital Human Research Ethic Committee (2020/13). Written informed consent was obtained from each patient. Declaration of Helsinki principles were followed in this study.

Definition of post Covid-19: Post-acute COVID-19 encompassed the patients who recovered from 3 weeks to 2 months earlier and with no prior heart disease. The patients had normal structural heart and normal cardiac troponin levels.

The patients with post-acute COVID-19 were divided into two groups according to the existence of symptoms. Group 1 was asymptomatic group (40 patients) and group 2 was symptomatic (65 patients).

Symptomatic patient was defined as an individual with suddenly new raised palpitation complaint with/without supraventricular arrhythmia (atrial tachycardia, sinus tachycardia) in 24 hours Holter analysis and/or fatigue association with exercise in the post COVID-19 period.

A comparison of two groups was made using biochemical parameters (NT-proBNP, Troponin I, and oxidative stress tests including nitric oxide), and imaging techniques (echocardiography, cardiovascular magnetic resonance, and cardiac positron emission tomography).

The patients with systolic dysfunction $(<50 \%$ ejection fraction), acute coronary syndrome, high troponin levels, renal failure, atrial fibrillation, pneumonia or pulmonary embolism were excluded.

Randomized fifteen patients underwent cardiovascular magnetic resonance (CMR) for tissue characterization evaluation (scar, fat, and edema). Because of normal CMR findings but high NT-proBNP, five of these patients underwent cardiac positron emission tomography (PET) for quantification of specific processes at the molecular level, such as metabolism, inflammation. ${ }^{10,11}$

\section{Measurement of NT-proBNP Plasma Levels}

NT-proBNP was measured by collecting $5 \mathrm{~mL}$ of whole blood into tubes containing potassium ethylene diamine tetra-acetic acid ( $1 \mathrm{mg} / \mathrm{mL}$ blood) upon admission. Plasma NT-proBNP was measured using the Immulite NT-proBNP assay $(\mathrm{pg} / \mathrm{mL})$.

\section{Oxidative Stress Assays and Oxidative Stress Markers}

The analyses of serum biochemical oxidative stress markers parameters were performed on an auto analyzer (Mindray BS 300) using commercial kits (RelAssay Diagnostic, Turkey). Venous blood samples were collected from all of the patients. Total antioxidant status (TAS, mmol Trolox Eq/L), total oxidant status (TOS, mmol $\mathrm{H}_{2} \mathrm{O}_{2} \mathrm{Eq} / \mathrm{L}$ ), oxidative stress index (OSI-TOS/TAS), paraoxonase $(\mathrm{PON}, \mathrm{U} / \mathrm{L})$, total thiol $(\mu \mathrm{mol} / \mathrm{L})$, serum native thiol $(\mu \mathrm{mol} / \mathrm{L})$, nitric oxide $(\mathrm{NO}, \mu \mathrm{mol} / \mathrm{L})$, and disulfide levels were measured for subjects. ${ }^{12,13}$

\section{Echocardiographic Evaluation}

Standard TTE was performed in the left lateral decubitus position using Philips Affiniti 50G device (Philips, USA) with a $2.5 \mathrm{MHz}$ transducer to examine two-dimensional images (2-D) and evaluate M-mode and Doppler values. In operation, depth was $20 \mathrm{~cm}$ and dynamic range was 6 . 
M-mode and 2-D images of the left ventricle and Doppler parameters were recorded on the basis of the American Society of Echocardiography and the European Association of Cardiovascular Imaging. ${ }^{14}$

Echocardiographic involvement was accepted as pericardial effusion or focal perimyocardial echo bright appearance. $^{15}$

\section{Cardiac MR Scan (CMR) and Cardiac Positron Emission Tomography (PET)}

To evaluate for myocarditis, cardiac magnetic resonance imaging was done using clinical 3-T scanners (SIGNA ${ }^{\mathrm{TM}}$ Voyager - $70 \mathrm{~cm}$ - GE Healthcare, USA). Conventional sequences were used for the acquisition of cardiac function, volumes, mass, and scar imaging. Myocardial T1 and T2 mapping were acquired in a single midventricular short-axis slice, while for T2 mapping, a validated sequence for measurement of myocardial edema was used. Late gadolinium enhancement (LGE) imaging was completed approximately 10 minutes after administration of $0.1 \mathrm{mmol} / \mathrm{kg}$ of body weight of gadobutrol (Gadovist; Bayer).

To assess for possible myocardial involvement, following a period of prolonged fasting ( $>10$ hours), the patients received 10-12 millicuries of $18 \mathrm{~F}-\mathrm{FDG}$ intravenously. 18F-FDG PET imaging with GE Discovery IQ was started after an uptake period of 90 minutes during which patients were asked to rest quietly (not to talk or engage in physical activity). The heart position was localized with a scout image (computerized tomography or radionuclide). Transmission scan (computerized tomography or radionuclide) was obtained for the measurement and correction of soft tissue attenuation followed by a non-gated cardiac 18F-FDG image acquisition (3-D mode). The cardiac emission images were reconstructed with and without attenuation correction.

\section{Statistical Analysis}

SPSS for Windows 23 (IBM Inc., Chicago, IL, USA) was used for statistical analysis. The normal distribution of the data was checked with Kolmogorov-Smirnov test. The comparison of categorical data was analyzed with Chi square test, while continuous parameters were compared with Independent Samples T-test. Continuous data were expressed as the mean $\pm \mathrm{SD}$ and categorical variables were presented as a number and percentage. In addition, we determined a proper cutoff value for BNP level through a ROC analysis and the areas under the ROC curves (AUCs) were calculated. A value of $p<0.05$ was considered as significant.

\section{Results}

Table 1 shows the demographic factors and clinical features of the patients. There was no significant statistical difference between the two groups regarding age, weight, and ejection fraction ( $p>0.05$; Table 1$)$. While the mean age of the patients in asymptomatic group (group 1) was $38.43 \pm 7.21$, that of the patients in symptomatic (group 2) was $37.35 \pm 7.01 \quad(\mathrm{p}>0.05)$. The patients in group 1 weighted $78.95 \pm 3.11 \mathrm{~kg}$, while the patients in group 2 weighed $78.72 \pm 3.65 \mathrm{~kg}$. In terms of ejection fraction (EF), EF in Group 1 was 57.27 \pm 1.67 , and EF in Group 2 was $57.38 \pm 1.46(\mathrm{p}>0.05)$.

Symptomatic patients had sinus tachycardia resting ECG or atrial tachycardia in 24 hours Holter analysis. Asymptomatic patients had normal sinus rhythm.

Biochemical parameters (NT-proBNP levels and oxidative stress markers):

Troponin I level and renal function tests of all patients were normal.

The symptomatic patients with post-acute COVID-19 syndrome had higher NT-proBNP levels than asymptomatic post COVID-19 patients (132.30 $\pm 35.15 ; 76.86$ \pm 16.79 , respectively; $\mathrm{p}<0.001)$. The symptomatic patients

Table I Demographic Features Between Two Groups Regarding Age, Weight, and Ejection Fraction

\begin{tabular}{|l|l|l|l|l|}
\hline & Asymptomatic & Symptomatic & Test Value & $\mathbf{p}$ \\
\hline Age & $38.43 \pm 7.21$ & $37.35 \pm 7.01$ & $0.742^{\mathrm{a}}$ & 0.453 \\
\hline Gender & $15(30.0)$ & $35(70.0)$ & $2.653^{\mathrm{b}}$ & 0.103 \\
\hline Weight (kilograms) & $25(45.5)$ & $30(54.5)$ & & $0.291^{\mathrm{a}}$ \\
\hline Ejection fraction & $78.95 \pm 3.11$ & $78.72 \pm 3.65$ & & 0.772 \\
\hline
\end{tabular}

Notes: ${ }^{\mathrm{a}}$ t-test; ${ }^{\mathrm{b}} \mathrm{Chi}$-squared test. 
with post -acute COVID-19 syndrome had lower NO levels than asymptomatic post COVID-19 patients (9.20 $\pm 3.08 ; 16.15 \pm 6.02$, respectively; $p<0.001$ ) (Table 2 ). No statistically significant difference was found between groups regarding other oxidative stress markers $(\mathrm{p}>0.05)$.

NO levels in patients $>125$ NT-proBNP are lower than the patients $<125$ NT-proBNP $(8.4 \pm 2.84 ; 14.13 \pm 5.81$, respectively; $\mathrm{p}<0.001$ ) (Table 3 ). There was no statistically significant difference between groups regarding other oxidative stress markers $(\mathrm{p}>0.05)$.

The cutoff value of NT-proBNP for symptomatic patients is $98.5 \mathrm{pg} / \mathrm{mL}$ (sensitivity $86.2 \%$ and specificity $90.0 \%$ ). Moreover, in the patients with $>98.5 \mathrm{pg} / \mathrm{mL}$ in NT-proBNP values, NO levels are significantly lower (9.34 $\pm 3.28 ; 15.91 \pm 6.12$, respectively; $\mathrm{p}<0.001)$.

\section{Imaging Techniques}

Echocardiographic evaluation: Echocardiographic measurements were performed for all of the patients. Left ventricular systolic and diastolic functions of the patients were normal. We only saw focal ecobright appearance and mild pericardial effusion in 2 patients within the 65 in the symptomatic group.

CMR evaluation: Fifteen patients underwent CMR. We did not see myocardial edema or patchy scarring for myocardial involvement on LGE images. Those patients had $>125$ NT proBNP levels (Figure 1A).

Cardiac PET evaluation: Five patients underwent cardiac PET (within the 15 patients who underwent CMR). We saw regional increased 18F-FGD uptake on myocardial jeopardized areas in five patients (Figure 1B).
Table 3 Oxidative Stress Markers According to NT-proBNP Levels

\begin{tabular}{|l|l|l|l|l|}
\hline & $\begin{array}{l}\text { NT-proBNP<1 25 } \\
(\mathbf{n}=\mathbf{6 9})(\mathbf{M} \pm \mathbf{S D})\end{array}$ & $\begin{array}{l}\text { NT-proBNP>1 25 } \\
(\mathbf{n}=\mathbf{3 6})(\mathbf{M} \pm \mathbf{S D})\end{array}$ & $\mathbf{t}$ & $\mathbf{p}$ \\
\hline TAS & $1.64 \pm 0.28$ & $1.61 \pm 0.24$ & 0.472 & 0.638 \\
\hline TOS & $4.77 \pm 1.22$ & $5.28 \pm 1.15$ & -1.645 & 0.105 \\
\hline OSI & $2.93 \pm 0.73$ & $3.29 \pm 0.70$ & -1.959 & 0.055 \\
\hline PON & $383.61 \pm 212.17$ & $446.45 \pm 320.34$ & -0.916 & 0.364 \\
\hline TTL & $252.55 \pm 59.87$ & $261.60 \pm 87.73$ & -0.479 & 0.634 \\
\hline NTL & $195.50 \pm 35.38$ & $208.68 \pm 58.34$ & -1.098 & 0.277 \\
\hline Disulfide & $28.92 \pm 22.39$ & $26.46 \pm 28.13$ & 0.382 & 0.704 \\
\hline NO & $14.13 \pm 5.81$ & $8.49 \pm 2.84$ & 4.589 & $<0.001$ \\
\hline
\end{tabular}

Abbreviations: NT-BNP, NT-proBNP (pg/mL); TAS, total antioxidant status (mmol Trolox Eq/L); TOS, total oxidant status ( $\mathrm{mmol} \mathrm{H}_{2} \mathrm{O}_{2} \mathrm{Eq} / \mathrm{L}$ ); OSI, oxidative stress index (TOS/TAS); PON, Paraoxonase (U/L); TTL, total thiol ( $\mu \mathrm{mol} / \mathrm{L})$ ) NTL, serum native thiol $(\mu \mathrm{mol} / \mathrm{L})$; $\mathrm{NO}$, nitric oxide $(\mu \mathrm{mol} / \mathrm{L})$.

\section{Discussion}

Post-acute COVID-19 syndrome has persistent symptoms after recovering from disease. Palpitations, fatigue, chest pain, and back pain are the most common symptoms in those patients. Viral infiltration, inflammation and microthrombi, and down-regulation of ACE-2 receptors are the likely mechanism. ${ }^{16,17}$

NT-proBNP is a natriuretic peptide synthesized and secreted by myocytes as a response to myocytes stretched by pressure overload or volume expansion of the ventricle. ${ }^{18}$ Moreover, cardiomyocyte damage or hypoxia may also cause the activation of NT-proBNP. ${ }^{19}$ We examined the patients with post-acute COVID-19 by making

Table 2 NT-proBNP Levels and Oxidative Stress Markers in Asymptomatic and Symptomatic Patients

\begin{tabular}{|l|l|l|l|l|}
\hline & Asymptomatic $(\mathbf{n}=\mathbf{4 0})(\mathbf{M} \pm \mathbf{S D})$ & Symptomatic $(\mathbf{n}=\mathbf{6 5}) \mathbf{( M \pm S D})$ & $\mathbf{t}$ & $\mathbf{p}$ \\
\hline NT-BNP & $76.86 \pm 16.79$ & $132.30 \pm 35.15$ & -9.327 & $<0.001$ \\
\hline TAS & $1.70 \pm 0.26$ & $1.60 \pm 0.27$ & 1.469 & 0.147 \\
\hline TOS & $4.99 \pm 1.44$ & $4.97 \pm 1.08$ & 0.071 & 0.944 \\
\hline OSI & $2.94 \pm 0.80$ & $3.14 \pm 0.70$ & -1.004 & 0.320 \\
\hline PON & $351.09 \pm 197.50$ & $439.79 \pm 285.86$ & -1.266 & 0.211 \\
\hline TTL & $253.76 \pm 73.93$ & $257.57 \pm 71.94$ & -0.195 & 0.846 \\
\hline NTL & $187.47 \pm 36.32$ & $207.95 \pm 49.58$ & -1.669 & 0.100 \\
\hline Disulfide & $33.59 \pm 25.77$ & $24.81 \pm 23.79$ & 1.349 & 0.182 \\
\hline NO & $16.15 \pm 6.02$ & $9.20 \pm 3.08$ & 6.138 & $<0.001$ \\
\hline
\end{tabular}

Abbreviations: NT-BNP, NT-proBNP (pg/mL); TAS, total antioxidant status (mmol Trolox Eq/L); TOS, total oxidant status (mmol $\mathrm{H}_{2} \mathrm{O}_{2}$ Eq/L); OSI, oxidative stress index (TOS/TAS); PON, Paraoxonase (U/L); TTL, total thiol ( $\mu \mathrm{mol} / \mathrm{L}) ; \mathrm{NTL}$, serum native thiol $(\mu \mathrm{mol} / \mathrm{L})$; NO, nitric oxide $(\mu \mathrm{mol} / \mathrm{L})$. 

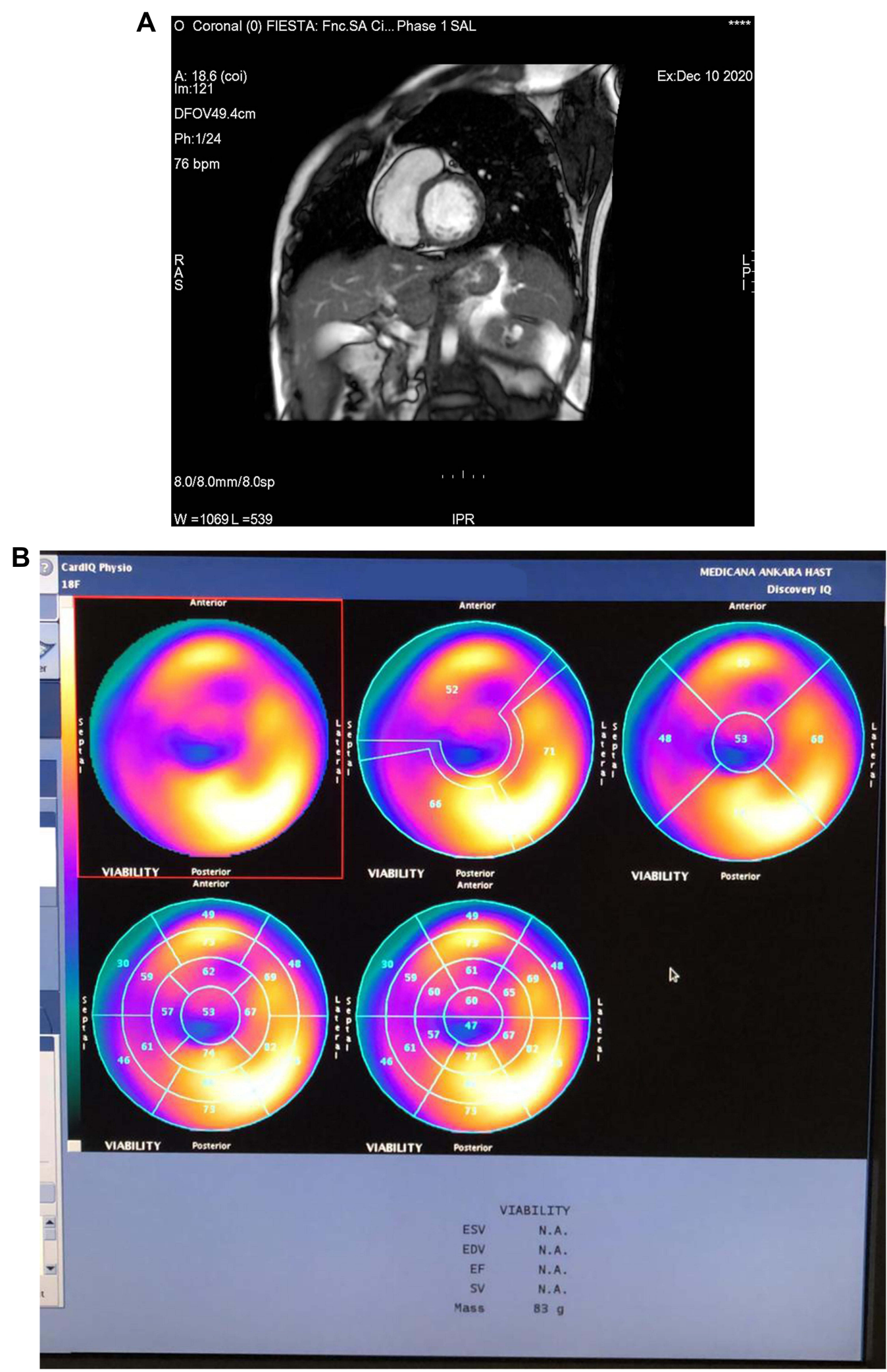

Figure I Cardiac magnetic resonance and cardiac PET imaging (same patient). (A) In short axis view, no cardiac involvement on LGE images. (B) Increased I8F-FDG-PET uptake on lateral and inferolateral walls of left ventricle on bulls eye map and on slices (indicating jeopardized area). 
blood analyses and imaging investigations (including echocardiography, CMR, cardiac PET). The patients with symptoms of post-acute COVID-19 syndrome had higher NT-proBNP levels than asymptomatic post COVID-19 patients $\quad(132.30 \pm 35.15 ; \quad 76.86 \pm 16.79, \quad$ respectively; $\mathrm{p}<0.001$ ). According to ESC guidelines, the upper limit of normal in the non-acute condition for NT-proBNP is $125 \mathrm{pg} / \mathrm{mL}$, while in acute condition, the cutoff value for NT-proBNP is $300 \mathrm{pg} / \mathrm{mL}^{20}{ }^{20}$ However, American guidelines do not recommend any cutoff levels for NT-proBNP in the chronic period. ${ }^{21}$ We have established that the cutoff value of NT-proBNP for symptomatic patients was 98.5 $\mathrm{pg} / \mathrm{mL}$ (sensitivity $86.2 \%$ and specificity $90.0 \%$ ) in ROC curves (Figure 1).

Interestingly, the symptomatic patients with post-acute COVID-19 syndrome had lower NO levels than asymptomatic post COVID-19 patients $(9.20 \pm 3.08 ; 16.15 \pm 6.02$, respectively; $\mathrm{p}<0.001)$. In the patients with $>125 \mathrm{pg} / \mathrm{mL}$ NT-proBNP levels, NO levels are significantly lower than the patients with $<125 \mathrm{pg} / \mathrm{mL}$ NT-proBNP levels (8.49 $\pm 2.84 ; 14.13 \pm 5.81$, respectively; $\mathrm{p}<0.001)$. Nitric oxide produced by endothelial cells is a strong vasodilatory and anti-inflammatory signaling molecule. Endothelial dysfunction is termed as a reduced capacity for nitric oxide production or/and decreased nitric oxide sensitivity. This condition causes a procoagulant and proinflammatory situation. $^{22}$ The findings of the present study support that thromboembolic events after acute period COVID-19 could be related to endothelial dysfunction.

ECG abnormality (sinus tachycardia/atrial tachycardia), elevated NT-proNBP levels, normal troponin I levels, normal LVEF in echocardiography, no infarction or infiltration on CMR, and increased regional 18F-FDG uptake on cardiac PET have been thought as myocardial fatigue. $^{23}$ Inflammatory state and decreased NO levels result in endothelial dysfunction. Then endothelial dysfunction causes heart failure symptoms (palpitations, exercise-related fatigue) such as heart failure with preserved ejection fraction (HFpEF). ${ }^{24}$ All combined, it can be suggested that the possible cardiac involvement mechanism of post-acute COVID-19 could be associated with myocardial fatigue and low NO levels (Figure 2). Duerr et al. reported another parameter to predict COVID-19-induced myocardial injury and mortality including availability of pericardial effusion and high CD8 (Killer) T cells/Treg/monocyte ratio. $^{25}$ Additionally, Lindner et al. analyzed COVID-19 autopsy cases and reported that viral presence within the myocardium could be documented, but not associated with an influx of inflammatory cells. ${ }^{26}$
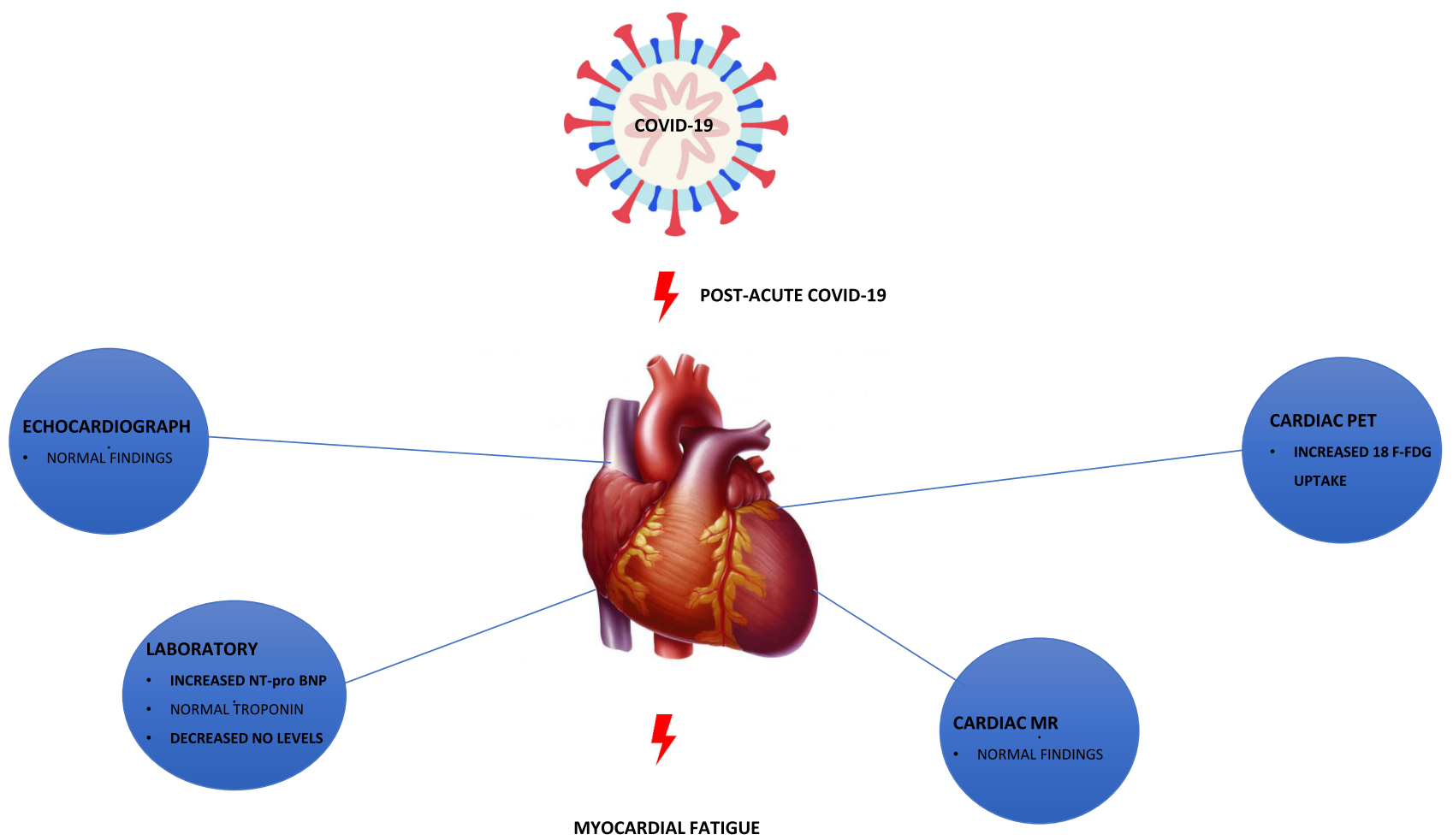

Figure 2 Effect of biochemical and imaging tests on possible cardiac involvement of post-acute COVID-19. 
Fifteen patients underwent CMR. We did not see myocardial edema or patchy scarring for myocardial involvement on LGE images. The patients had $>125$ NT proBNP levels. However, five patients underwent cardiac PET (within the 15 patients who underwent CMR). We saw a regional increase in 18F-FGD uptake on myocardial jeopardized areas in these five patients, in spite of normal CMR findings.

This study showed that the patients with post-acute COVID-19 could have subtle myocardial involvement. Besides, these findings indicate subtle myocardial inflammation could be missed by the CMR in subacute/chronic myocarditis.

Although there were no evidence of myocardial damage after post-acute COVID-19 such as echocardiographic and cardiac enzyme abnormality, transient ECG abnormality, PET findings could be an evaluation of cardiac involvement. CMR could have low sensitivity for patients with subacute/chronic myocarditis. Therefore, cardiac PET could replace or add to CMR in suspected subacute/chronic myocarditis.

If endomyocardial biopsy is not possible, the diagnosis is of suspected diagnosis of myocarditis with or without associated pericarditis and can be supported by CMR according to Lake Louise criteria (LLC), which target three aspects of myocardial inflammation: edema, hyperemia, and necrosis and/or fibrosis. ${ }^{27}$ However, diffuse or subtle myocardial inflammation can be missed by the original LLC. Thus, the original LLC was revised with mapping techniques in the acute myocarditis diagnosis in $2018 .^{28}$ The patients with symptomatic COVID-19 and acute myocarditis were found to have a pattern of diffuse myocardial edema in CMR evaluation, ${ }^{29,30}$ but in following clinical change details of CMR are rather limited. ${ }^{31}$

However, in subacute/chronic period, CMR's limitation in diagnosing myocarditis is the evolving nature of myocardial edema and necrosis over time after the initial insult. $^{32}$ On the other hand, cardiac FDG-PET has a potential role in the assessment of myocarditis by detecting the underlying myocardial inflammatory activity, which is abnormally increased myocardial uptake. FDGPET findings may support complementary and incremental information to CMR for subtle or borderline myocarditis, and increasing specificity for chronic myocarditis.

A few limitations exist in our study. First, patients mostly consist of recovered COVID-19 from 3 weeks to 2 months ago and no prior heart disease. Second, NT-proBNP, Troponin I, oxidative stress tests were the only markers investigated in this study. This limitation did not affect the results. Studying with other cardiac inflammatory markers would emphasize advantages (e.g. efficacy, specificity) in prediction of fatigue. There is no cardiac biopsy procedure. Furthermore, this was a single-center study and additionally the small sample size may have posed limitations in this study.

\section{Conclusion}

We have informed elevated NT-proNBP levels, low serum NO levels, normal CMR findings and increased 18F-FDG uptake on cardiac PET in subacute/chronic term post-acute COVID-19 disease. The following of those patients with post-acute COVID-19 should also target the possibility of risk of heart failure.

\section{Abbreviations}

M, Mean; SD, Standard Deviation; NT-BNP, NT-proBNP (pg/mL); TAS, total antioxidant status (mmol Trolox Eq/ L); T.O.S, total oxidant status (mmol $\left.\mathrm{H}_{2} \mathrm{O}_{2} \mathrm{Eq} / \mathrm{L}\right)$; OSI, oxidative stress index; (TOS/TAS); PON, Paraoxonase (U/ $\mathrm{L})$; TTL, total thiol $(\mu \mathrm{mol} / \mathrm{L})$; NTL, serum native thiol $(\mu \mathrm{mol} / \mathrm{L})$; NO, nitric oxide $(\mu \mathrm{mol} / \mathrm{L})$; LGE, Late gadolinium enhancement imaging.

\section{Data Sharing Statement}

The data will not be shared. Due to the restrictions from Medicana International Ankara Hospital, the data are available upon request for any researcher based on a standard agreement on data provision.

\section{Ethics Approval and Consent to Participate}

The study protocol adopted the ethical guidelines of the 1975 Declaration of Helsinki and was approved by the Medicana International Ankara Hospital Human Research Ethic Committee (2020/13). Written and informed consent was obtained from each participant included in the study.

\section{Acknowledgments}

Atılım University Medical School, and Medicana International Ankara Hospital for support of biochemical and imaging tests.

\section{Author Contributions}

All authors made a significant contribution to the work reported, whether that is in the conception, study design, execution, acquisition of data, analysis and interpretation, 
or in all these areas; took part in drafting, revising or critically reviewing the article; gave final approval of the version to be published; have agreed on the journal to which the article has been submitted; and agree to be accountable for all aspects of the work.

\section{Funding}

This work was supported by Medicana International Ankara Hospital and Atilim University School of medicine. None of the funding sources had an influence on the design or production of the article.

\section{Disclosure}

The authors report no other potential conflicts of interest for this work and no financial or other relationships that might lead to a conflict of interest. There are no relationships with industry.

\section{References}

1. Wang D, Hu B, Hu C, et al. Clinical characteristics of 138 hospitalized patients with 2019 novel coronavirus-infected pneumonia in Wuhan, China. JAMA. 2020;323:1061-1069. doi:10.1001/ jama.2020.1585

2. Hendren NS, Drazner MH, Bozkurt B, Cooper LT Jr. Description and proposed management of the acute COVID-19 cardiovascular syndrome. Circulation. 2020;141:1903-1914. doi:10.1161/ CIRCULATIONAHA.120.047349

3. Perrin R, Riste L, Hann M, Walther A, Mukherjee A, Heald A. Into the looking glass: post-viral syndrome post COVID-19. Med Hypotheses. 2020;144:110055. doi:10.1016/j.mehy.2020.110055

4. Peretto G, Sala S, Rizzo S, et al. Ventricular arrhythmias in myocarditis: characterization and relationships with myocardial inflammation. J Am Coll Cardiol. 2020;75:1046-1057. doi:10.1016/ j.jacc.2020.01.036

5. Bowles KH, McDonald M, Barrón Y, Kennedy E, O'Connor M, Mikkelsen M. Surviving COVID-19 after hospital discharge: symptom, functional, and adverse outcomes of home health recipients. Ann Intern Med. 2021;174:316-325. doi:10.7326/M20-5206

6. Onodera M, Nakamura M, Tanaka F, et al. Plasma B-type natriuretic peptide is useful for cardiovascular risk assessment in community-based diabetes subjects: comparison with albuminuria. Int Heart J. 2012;53:176-181. doi:10.1536/ihj.53.176

7. Welsh P, Hart C, Papacosta O, et al. Prediction of cardiovascular disease risk by cardiac biomarkers in 2 United Kingdom cohort studies: does utility depend on risk thresholds for treatment? Hypertension. 2016;67:309-315. doi:10.1161/ HYPERTENSIONAHA.115.06501

8. Gao L, Jiang D, Wen XS, et al. Prognostic value of NT-proBNP in patients with severe COVID-19. Respir Res. 2020;21:83. doi:10.1186/s12931-020-01352-w

9. Fang W, Jiang J, Su L, et al. The role of NO in COVID-19 and potential therapeutic strategies. Free Radic Biol Med. 2021;163:153-162. doi:10.1016/j.freeradbiomed.2020.12.008

10. Bailey DL, Antoch G, Bartenstein P, et al. Combined PET/MR: the real work has just started. Summary report of the Third International Workshop on PET/MR Imaging; February 17-21, 2014, Tübingen, Germany. Mol Imaging Biol. 2015;17:297-312. doi:10.1007/s11307014-0818-0
11. Hendel RC, Berman DS, Di Carli MF, et al. ACCF/ASNC/ ACR/ AHA/ASE/SCCT/SCMR/SNM Appropriate Use Criteria for Cardiac Radionuclide Imaging: a Report of the American College of Cardiology Foundation Appropriate Use Criteria Task Force, the American Society of Nuclear Cardiology, the American College of Radiology, the American Heart Association, the American Society of Echocardiography, the Society of Cardiovascular Computed Tomography, the Society for Cardiovascular Magnetic Resonance, and the Society of Nuclear Medicine. J Am Coll Cardiol. 2009;53:2201-2229.

12. Kilic Y, Ozer A, Tatar T, et al. Effect of picroside II on hind limb ischemia reperfusion injury in rats. Drug Des Devel Ther. 2017;11:1917-1925. doi:10.2147/DDDT.S132401

13. Kucuk A, Polat Y, Kilicarslan A, et al. Irisin protects against hind limb ischemia reperfusion injury. Drug Des Devel Ther. 2021;15:361-368. doi:10.2147/DDDT.S279318

14. Lang RM, Badano LP, Mor-Avi V, et al. Recommendations for cardiac chamber quantification by echocardiography in adults: an update from the American Society of Echocardiography and the European Association of Cardiovascular Imaging. $J \mathrm{Am} S o \mathrm{c}$ Echocardiogr. 2015;28:1-39.e14. doi:10.1016/j.echo.2014.10.003

15. Saricam E, Saglam Y, Hazirolan T. Clinical evaluation of myocardial involvement in acute myopericarditis in young adults. $B M C$ Cardiovasc Disord. 2017;17:129. doi:10.1186/s12872-017-0564-8

16. Kochi AN, Tagliari AP, Forleo GB, Fassini GM, Tondo C. Cardiac and arrhythmic complications in patients with COVID-19. J Cardiovasc Electrophysiol. 2020;31:1003-1008. doi:10.1111/ jce. 14479

17. Madjid M, Safavi-Naeini P, Solomon SD, Vardeny O. Potential effects of coronaviruses on the cardiovascular system: a review. JAMA Cardiol. 2020;5:831-840. doi:10.1001/jamacardio.2020.1286

18. Del Ry S, Cabiati M, Clerico A. Natriuretic peptide system and the heart. Front Horm Res. 2014;43:134-143.

19. Volpe M, Rubattu S, Burnett J. Natriuretic peptides in cardiovascular diseases: current use and perspectives. Eur Heart $J$. 2014;35:419-425. doi:10.1093/eurheartj/eht466

20. Ponikowski P, Voors AA, Anker SD, et al. 2016 ESC Guidelines for the diagnosis and treatment of acute and chronic heart failure: the Task Force for the diagnosis and treatment of acute and chronic heart failure of the European Society of Cardiology (ESC). Developed with the special contribution of the Heart Failure Association (HFA) of the ESC. Eur J Heart Fail. 2016;18:891-975.

21. Yancy CW, Jessup M, Bozkurt B, et al. 2013 ACCF/AHA guideline for the management of heart failure: a report of the American College of Cardiology Foundation/American Heart Association Task Force on Practice Guidelines. J Am Coll Cardiol. 2013;62:e147-e239. doi:10.1016/j.jacc.2013.05.019

22. Cyr RA, Huckaby LV, Shiva SS, Zuckerbraun BS. Nitric oxide and endothelial dysfunction. Crit Care Clin. 2020;36:307-321. doi:10.1016/j.ccc.2019.12.009

23. Banerjee P. Heart failure: a story of damage, fatigue and injury? Open Heart. 2017;4:e00684. doi:10.1136/openhrt-2017-000684

24. Giamouzis G, Schelbert EB, Butler J. Growing evidence linking microvascular dysfunction with heart failure with preserved ejection fraction. $J$ Am Heart Assoc. 2016;5:e03259. doi:10.1161/ JAHA.116.003259

25. Duerr GD, Heine A, Hamiko M, et al. Parameters predicting COVID-19-induced myocardial injury and mortality. Life Sci. 2020;260:118400. doi:10.1016/j.lfs.2020.118400

26. Lindner D, Fitzek A, Bräuninger $\mathrm{H}$, et al. Association of cardiac infection with SARS-CoV-2 in confirmed COVID-19 autopsy cases. JAMA Cardiol. 2020;5(11):1281-1285. doi:10.1001/ jamacardio.2020.3551

27. Friedrich MG, Sechtem U, Schulz-Menger J, et al. Cardiovascular magnetic resonance in myocarditis: a JACC white paper. $J$ Am Coll Cardiol. 2009;53:1475-1487. doi:10.1016/j.jacc.2009.02.007 
28. Ferreira VM, Schulz-Menger J, Holmvang G, et al. Cardiovascular magnetic resonance in nonischemic myocardial inflammation: expert recommendations. $J \mathrm{Am}$ Coll Cardiol. 2018;72:3158-3176. doi:10.1016/j.jacc.2018.09.072

29. Luetkens JA, Isaak A, Oztürk C, et al. Cardiac MRI in Suspected Acute COVID-19 Myocarditis. Radiol Cardiothorac Imaging. 2021;3 (2):e200628. doi:10.1148/ryct.2021200628

30. Luetkens JA, Isaak A, Zimmer S, et al. Diffuse myocardial inflammation in COVID-19 associated myocarditis detected by multiparametric cardiac magnetic resonance imaging. Circ Cardiovasc Imaging. 2020;13 (5):e010897. doi:10.1161/CIRCIMAGING.120.010897
31. Mishra AK, Lal A, Sahu KK, Kranis M, Sargent J. Quantifying and reporting cardiac findings in imaging of COVID-19 patients. Monaldi Arch Chest Dis. 2020;90(4). doi:10.4081/monaldi.2020.1394

32. de Roos A. Diagnosis of myocarditis at cardiac MRI: the continuing quest for improved tissue characterization. Reviews and commentary. Radiology. 2019;292:618-619. doi:10.1148/radiol.2019191476

\section{Publish your work in this journal}

The International Journal of General Medicine is an international, peer-reviewed open-access journal that focuses on general and internal medicine, pathogenesis, epidemiology, diagnosis, monitoring and treatment protocols. The journal is characterized by the rapid reporting of reviews, original research and clinical studies across all disease areas. The manuscript management system is completely online and includes a very quick and fair peer-review system, which is all easy to use. Visit http://www.dovepress.com/ testimonials.php to read real quotes from published authors.

Submit your manuscript here: https://www.dovepress.com/international-journal-of-general-medicine-journal 